\title{
Gastric Subserous Vaccination With Helicobacter pylori Vaccine: An Attempt to Establish Tissue-Resident CD4+ Memory T Cells and Induce Prolonged Protection
}

\section{OPEN ACCESS}

Edited by:

Nicholas J. Mantis,

Wadsworth Center, United States

Reviewed by:

Karl Kai McKinstry,

University of Central Florida,

United States

Tara Marlene Strutt,

University of Central Florida,

United States

*Correspondence:

Tao Xi

xitao18@hotmail.com

Yingying Xing

cpuskyxyy@126.com

Specialty section:

This article was submitted to Immunological Memory,

a section of the journal

Frontiers in Immunology

Received: 30 October 2018

Accepted: 01 May 2019

Published: 17 May 2019

Citation:

Liu W, Zeng Z, Luo S, Hu C, Xu N,

Huang A, Zheng L, Sundberg EJ, Xi T and Xing $Y$ (2019) Gastric Subserous Vaccination With Helicobacter pylori Vaccine: An Attempt to Establish Tissue-Resident CD4+ Memory T

Cells and Induce Prolonged

Protection. Front. Immunol. 10:1115.

doi: 10.3389/fimmu.2019.01115

\begin{abstract}
Wei Liu ${ }^{1,2}$, Zhiqin Zeng ${ }^{1,2}$, Shuanghui Luo ${ }^{1,2}$, Chupeng $\mathrm{Hu}^{1,2}$, Ningyin $\mathrm{Xu}^{1,2}$, An Huang ${ }^{1,2}$, Lufeng Zheng ${ }^{1,2}$, Eric J. Sundberg ${ }^{3,4}$, Tao $\mathrm{Xi}^{1,2 \star}$ and Yingying Xing ${ }^{1,2 \star}$

${ }^{1}$ School of Life Science and Technology, China Pharmaceutical University, Nanjing, China, ${ }^{2}$ Jiangsu Key Laboratory of Carcinogenesis and Intervention, China Pharmaceutical University, Nanjing, China, ${ }^{3}$ Institute of Human Virology, Department of Medicine, University of Maryland School of Medicine, Baltimore, MD, United States, ${ }^{4}$ Department of Microbiology and Immunology, University of Maryland School of Medicine, Baltimore, MD, United States
\end{abstract}

Tissue-resident memory $\mathrm{T}$ (Trm) cells are enriched at the sites of previous infection and required for enhanced protective immunity. However, the emergence of Trm cells and their roles in providing protection are unclear in the field of Helicobacter pylori $(H$. pylori) vaccinology. Here, our results suggest that conventional vaccine strategies are unable to establish a measurable antigen (Ag)-specific memory cell pool in stomach; in comparison, gastric subserous injection of mice with micro-dose of Alum-based $\mathrm{H}$. pylori vaccine can induce a pool of local CD4+ Trm cells. Regional recruitment of Ag-specific CD4+ T cells depends on the engagement of Ag and adjuvant-induced inflammation. Prior subcutaneous vaccination enhanced this recruitment. A stable pool of Ag-specific CD4+ T cells can be detected for 240 days. Two weeks of FTY720 administration in immune mice suggests that these cells do not experience the recirculation. Immunohistochemistry results show that close to the vaccination site, abundant CD4+T cells locate on epithelial niches, independent of lymphocyte cluster. Paradigmatically, Ag-specific CD4+ T cells with a phenotype of CD69+CD103-are preferential on lymphocytes isolated from epithelium. Upon Helicobacter infection, CD4+ Trm cells orchestrate a swift recall response with the recruitment of circulating antigen-specific Th1/Th17 cells to trigger a tissue-wide pathogen clearance. This study investigates the vaccine-induced gastric CD4+ Trm cells in a mice model, and highlights the need for designing a vaccine strategy against $H$. pylori by establishing the protective CD4+ Trm cells.

Keywords: tissue-resident memory T cells, CD4+ T cells, subunit vaccine, Helicobacter pylori, immunological memory

\section{INTRODUCTION}

In addition to effector memory $\mathrm{T}(\mathrm{Tem})$ cells and central memory $\mathrm{T}(\mathrm{Tcm})$ cells, tissue-resident memory $\mathrm{T}(\mathrm{Trm})$ cells are the third subset of memory $\mathrm{T}$ cells that reside in the non-lymphoid tissues without entering recirculation (1). Proximity to the entry points of pathogens and their state of differentiation ensure that Trm cells can rapidly react to local infection (2-5). A study 
reveals that a subset of effector $\mathrm{T}$ cells within non-inflamed tissues manipulate the potential to differentiate into Trm cells after adaption to local survival cues (6). However, in most scenarios of immunization/infection, Trm cells emerged after the resolution of local inflammation $(1,7)$. Trm cell populations are well-characterized in terms of Trm cells derived from CD8+ T cells or generated in response to invasive pathogens, but are less well-understood in terms of Trm cells derived from CD4+ T cells or generated in response to non-invasive pathogenic bacteria (3).

Helicobacter pylori (H. pylori) is a highly successful pathogen that colonizes the stomach of humans (8). Development of vaccines is one of the desirable alternative strategies to eliminate the threat of $H$. pylori. Previous clinical trials have demonstrated that many attempts fail to provide sufficient protection against $H$. pylori in human $(9,10)$. Evidence obtained from mice suggests a strong ability of this bacterium to alter the detection of pattern recognition receptors (PRRs) and subvert host immune system by producing multiple virulence factors (11). When facing this pathogen, host immune system is unable to orchestrate a potent response to purge the infection. Most infected individuals develop asymptomatic chronic gastritis, which sustains over their lifetimes if no antibiotic intervention. It is commonly accepted the need for $\mathrm{CD} 4+\mathrm{T}$ cells, rather than $\mathrm{CD} 8+\mathrm{T}$ cells or antibody-mediated responses, in providing protection $(12,13)$. Multiple studies using conventional vaccine strategies show that vaccination reduces $H$. pylori colonization in mice (13-18). Yet, the emergence of gastric Trm cells in these studies remains enigmatic. Dependence solely on recalling circulating memory $\mathrm{T}$ cells induced by conventional vaccination may result in a delay and "miss the boat" for optimal protection. Establishing a CD4+ Trm pool in stomach by vaccination and exploring the generation, maintenance, and behavior of these cells are attractive. However, the first-line challenges are how to send these pathogen-specific CD4+ T cells into the tissue "battlefield" and make sure that a CD4+ Trm pool can be detected. To address these gaps in the field, by using intracellular cytokine staining, we assessed the magnitude of antigen (Ag)-specific CD4+ cells after various vaccinations and found a measurable pool of Agspecific CD4+ Trm cells in mice that vaccinated with microdose of Alum-based $H$. pylori vaccine in gastric subserosa layer (GSL). The characteristics and mechanism of protection against H. pylori were further investigated in these cells. This study proposes a notion that investigators should take into account a subset of Trm cells when planning an $H$. pylori vaccine strategy.

\section{MATERIALS AND METHODS}

\section{Vaccine Preparation}

Purified CCF protein and GEM particles were prepared and stored according to previous protocols $(19,20)$. Briefly, the CCF protein was expressed by Escherichia coli Rosetta (DE3) cells with pET-28a-CCF. The protein was first purified by nickel affinity chromatography (GE Healthcare), followed by anionexchange chromatography with DEAE Sepharose FF (Amersham Pharmacia Biotech AB, Sweden). The purity of CCF was confirmed by Coomassie blue staining. The GEM particles were prepared by Lactococcus lactis NZ9000 cells using a hot-acid water bath. Vaccine with Alum was prepared with an equal volume of CCF solution and Alum adjuvant. CpG ODN 1826 was obtained from Sangon Biotech Co., Led. (China, Shanghai) and dissolved in CCF solution before intranasal vaccination.

\section{Animals and Immunizations}

Eight-week-old female C57BL/6J mice were obtained from the Comparative Medicine Center of Yangzhou University and bred at the China Pharmaceutical University Animal Experimental Center. All animal experiments were approved by the Animal Ethical and Experimental Committee of China Pharmaceutical University. The immunizations were performed according to the timetables in the figures and the doses of antigen and adjuvants are indicated in the figure captions or special region of the figure.

\section{Gastric Subserous Layer Vaccination}

Mice were anesthetized with $15 \mathrm{mg} / \mathrm{kg}$ Xylazine and $100 \mathrm{mg} / \mathrm{kg}$ Ketamine, and placed on a body temperature heating pad. After shaving the right abdomen, a $1.5 \mathrm{~cm}$ incision was made above the stomach. After laparotomy, the stomach was localized, and $5 \mu \mathrm{l}$ vaccine preparation (Volume, CCF solution: Alum $=1: 1$, containing $\sim 7.5 \mu \mathrm{g} \mathrm{CCF}$ ) was injected into the gastric subserous layer of the greater curvature using a Hamilton syringe with a $33 \mathrm{G}$ needle. Then, suturing with PGA absorbable sutures was performed using uninterrupted sutures for the peritoneum and interrupted sutures for the skin incision (Shanghai Pudong Jinhuan Medical Products Co., Ltd.).

\section{Preparation of Single-Cell Suspensions From Gastric Tissue}

Single-cell suspensions were prepared as a previous study with modifications (21). Briefly, the whole stomach was isolated, cut through the lesser curvature, and the contents were removed before being placed into $15 \mathrm{ml}$ RPMI 1640 containing $10 \mathrm{mM}$ HEPES, $10 \%$ FBS, $4 \mathrm{mM}$ EDTA, and $0.5 \mathrm{mM}$ dithiothreitol. Gastric epithelial lymphocytes were isolated by shaking at 250 $\mathrm{rpm}$ and $37^{\circ} \mathrm{C}$ for $30 \mathrm{~min}$. Tissues were then minced and incubated with another $15 \mathrm{ml}$ RPMI 1640 containing $10 \mathrm{mM}$ HEPES, $10 \%$ FBS, $4 \mathrm{mM}$ EDTA, and $0.5 \mathrm{mM}$ dithiothreitol for $15 \mathrm{~min}$ to isolate the remaining lymphocytes. Supernatants were passed through a $70 \mu \mathrm{m}$ cell strainer. After washing and centrifugation, cell pellets were resuspended in an appropriate medium for further analysis or culture.

\section{Preparation of Single-Cell Suspensions From Lymphoid Organs and Blood}

The spleen and mesenteric lymph nodes were isolated and gently pushed through a $70 \mu \mathrm{m}$ cell strainer. After extensive washing, cells from the lymph nodes were collected. The cells from the spleen and blood were suspended in $7 \mathrm{ml}$ erythrocyte lysis buffer (Biolegend) and washed twice with $10 \mathrm{ml}$ PBS containing 5\% FBS. Cells were collected for FACS analysis or stimulated in vitro.

\section{Antigen-Specific CD4+ T Cell Analysis}

Single-cell suspensions from the stomach were purified with $67 / 44 \%$ Percoll gradients. The cells at the interface were collected and washed with $7 \mathrm{ml}$ RPMI 1640 containing 10\% FBS. To detect 
Ag-specific CD4+ T cells, purified single-cell suspensions from the stomach, MLN, spleen or blood were stimulated with $1 \times 106$ naïve, CFSE-labeled splenocytes that were preloaded with CCF in RPMI 1640 containing 10\% FBS and $5 \mu \mathrm{g} / \mathrm{ml}$ BFA for $12 \mathrm{~h}$. After collection, cells were stained for intracellular cytokines.

\section{FACS Analysis}

For IFN- $\gamma$ and IL-17 intracellular cytokine staining, in vitro restimulated cells were first stained with anti-CD4 (GK1.5) and anti-CD90.2 (30-H12) antibodies, then fixed and permeabilized with Intracellular Staining Fixation/Permeabilization Wash Buffer (Biolegend, San Diego, CA) and stained intracellularly with anti-IFN- $\gamma$ (XMG1.2) and anti-IL-17 (9B10) antibodies. For cell phenotype detection, single-cell suspensions were stained with the following antibodies: anti-CD3E (145-2C11), antiCD90.2 (30-H12), anti-CD45 (30-F11), anti-CD4 (GK1.5 or RM4-4), anti-CD11b (M1/70), anti-CD8 $\alpha$ (53-6.7), anti-CD19 (6D5), anti-MHC class II (M5/114.15.2), anti-CD69 (H1.2F3), anti-CD25 (3C7), anti-CD44 (IM7), anti-CD103 (2E7), antiTCR $\gamma \delta$ (UC7-13D5), anti-Ly6C (HK1.4), anti-Gr-1 (RB6-8C5), anti-CD11c (N418), and anti-F4/80 (BM8) purchased from Biolegend or BD Pharmingen. Multiparameter analyses were performed on a BD FACS Aria II or a BD FACS Calibur flow cytometer.

\section{Immunofluorescent Histology}

For gastric histology, the longitudinal specimens were fixed with $4 \%$ paraformaldehyde, embedded in paraffin, and stained with hematoxylin and eosin (HE). For CD4 immunofluorescent staining, 20 or $10 \mu \mathrm{m}$ frozen sections were cut and dried at room temperature. After blocking, these sections were stained with an Alexa Fluor ${ }^{\circledR}$ 488-anti-CD4 (GK1.5, Biolegend) antibody and/or purified anti-CD11b (M1/70, Biolegend) or anti-CD8 $\alpha$ (53-6.7, Biolegend) antibody followed by goat anti-rat IgG2a/IgG2b Alexa Fluor ${ }^{\circledR}$ 488/594 antibody (Biolegend). The slides were washed and counterstained with DAPI to visualize cell nuclei, and images were acquired with a Panoramic 250 Flash III Scanner (3D Histech). The number of CD4+ cells in each section was counted in a $0.5 \mu \mathrm{m} \times 0.5 \mu \mathrm{m}$ area with highest CD4+ cell signaling.

\section{Quantitative RT-PCR}

Gastric RNA extraction and reverse transcription were carried out as described previously (22). PCR amplification was performed with a conventional TaqMan method. TaqMan gene primers and probes were designed by Sangon Biotech Co., Led. (China, Shanghai) based on the following sequence numbers: CCL5, Mm01302427_m1; CXCL9, Mm00434946_m1; CXCL10, Mm00445235_m1; GADPH Mm99999915_g1.

\section{FTY720 Treatment}

For FTY720 treatment, $1 \mathrm{mg} / \mathrm{kg}$ FTY720 was injected i.p. daily to block circulating memory $\mathrm{T}$ cell egress from the lymphoid nodes according to the design of the experiments.

\section{Neutralizing Antibody Experiments}

Immune mice were i.p. injected with $100 \mu \mathrm{g}$ anti-CD4 antibody (GK1.5, BioXcell), anti-RatIgG1, anti-IFN- $\gamma$ (XMG1.2, BioXcell) and anti-IL-17A (17F3, BioXcell) antibody every 2 days to deplete CD4+ T cells, IFN- $\gamma$ and IL-17A according to the design of experiments.

\section{H. pylori Challenge}

Helicobacter pylori SS1 was cultured as previously described (22). Sixty days after the last vaccination, the mice were challenged with $1 \times 10^{9} \mathrm{CFU} H$. pylori SS1 (determined by turbidimetry) by gavage in $200 \mu \mathrm{l}$ of $0.2 \%$ sodium bicarbonate solution.

\section{Quantitative Culture of $\boldsymbol{H}$. pylori}

Quantitative culture of $H$. pylori was performed as previously described (22). Briefly, half of the stomach was homogenized in $500 \mu \mathrm{l}$ Brain Heart Infusion (BHI) broth and plated at a series of dilutions on BHI plates. The bacterial colonization was calculated at the whole organ level.

\section{Statistics}

GraphPad Prism 7.0 software was used for statistical analyses. The differences between the groups were assessed using the Kruskal-Wallis test or Mann-Whitney $U$-test. $P<0.05$ was considered statistically significant.

\section{RESULTS}

\section{Ag-specific CD4+ Effector T Cells Are Present in Stomach After Conventional Vaccinations, but Fail to Give Rise to a Formidable CD4+ Memory T Cell Pool}

An outstanding question in the field of $H$. pylori vaccinology is whether conventional vaccinations can induce an Ag-specific $\mathrm{CD} 4+$ cell population in the stomach. Here, we used a recombinant $H$. pylori subunit vaccine, CCF, as a model Ag. CCF was constructed by multi-epitopes from $H$. pylori urease, and selfadjuvant regions from Salmonella typhimurium phase I flagellin FliC and cholera toxin B (20). To detect Ag-specific CD4+ T cells in stomach, we isolated total purified leukocytes from whole stomach of immune mice and co-cultured these cells with $1 \times 10^{6}$ Ag-preloaded, CFSE-labeled naive splenocytes for $12 \mathrm{~h}$ in the presence of Brefeldin A (BFA). Two crucial effector cytokines, IFN- $\gamma$ and IL-17A, for anti-H. pylori immunity were used to identify Ag-specific $\mathrm{T}$ cells. In the preliminary data, we found that for conventional vaccinations, Ag-specific CD4+ cells that produced only IFN- $\gamma$ or IL-17A were rare, but the combination of IFN- $\gamma$ and IL-17A allowed for the detection of more Ag-specific CD4+ T cells in these groups (Supplementary Figure 1).

Previous studies suggest that specific vaccinations can evoke a transient state that which allows Teff cell migration into nonlymphoid tissues at effector stage $(6,23)$. To detect gastric Ag-specific CD4+ T cells after conventional vaccinations, we performed different vaccine administrations on the mice and compared the gastric Ag-specific CD4+ T cells at Day 7 and Day 30 (Figures 1A,B). Naïve mice were used as a negative control to exclude non-specific staining, and mice receiving gastric subserosa layer (GSL) vaccination were used as a positive control. Ag-specific CD4+ T cells could be observed on Day 7 in stomach of mice receiving subcutaneous (s.c.), intranasal 
A
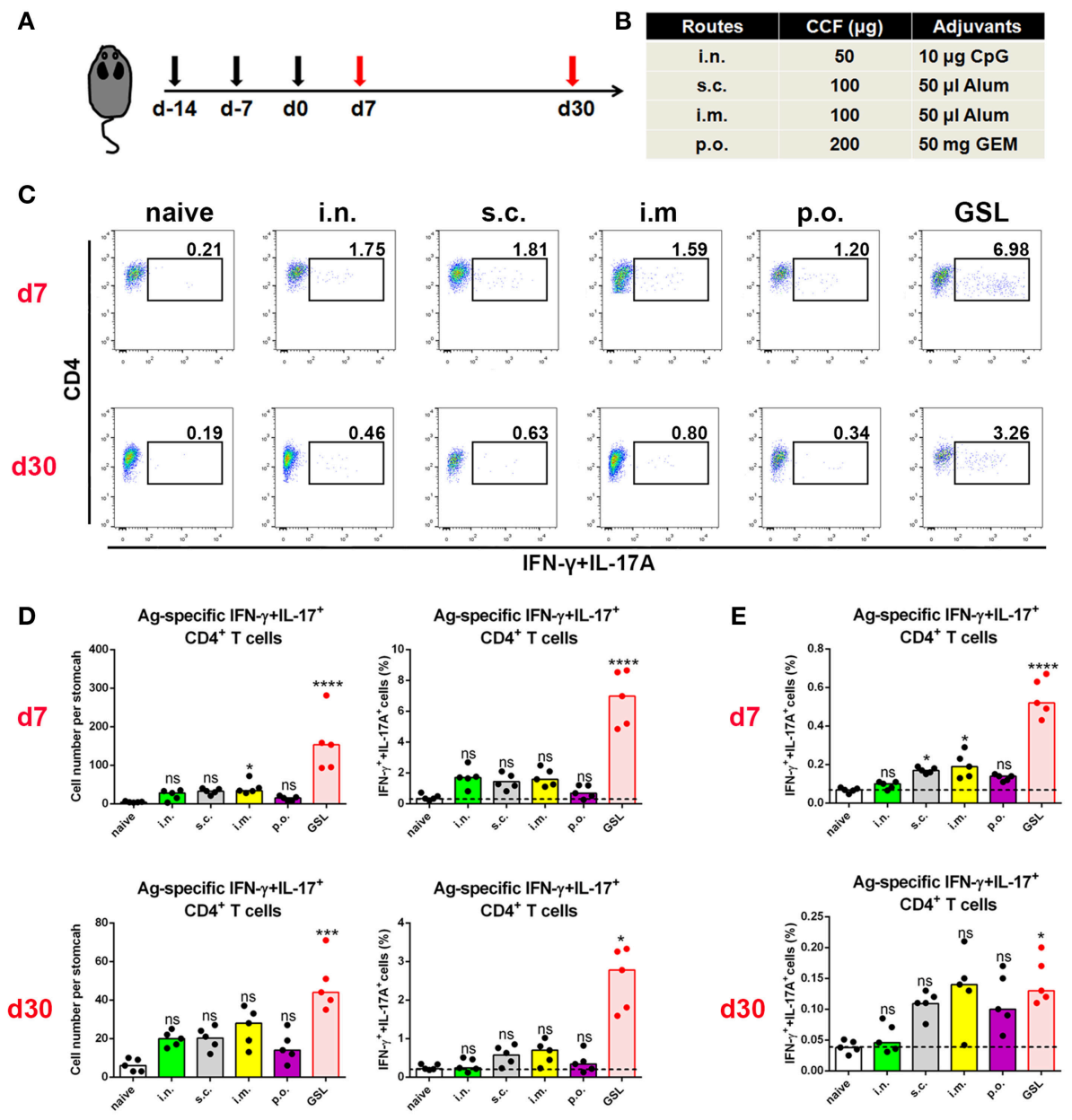

FIGURE 1 | Conventional vaccinations failed to induce a durable Ag-specific CD4+ memory T cell pool in the stomach. (A) C57BL/6J mice were immunized at Day-14, Day-7, and Day 0 with different vaccine strategies (B). At Day 7 and Day 30 after the last vaccination, mice were sacrificed and the Ag-specific CD4+ T cells in stomach were measured by intracellular cytokine staining. Purified cells were restimulated with Ag-preloaded, CFSE-labeled, naïve splenocytes for $12 \mathrm{~h}$ in the presence of $5 \mu \mathrm{g} / \mathrm{ml} \mathrm{BFA}$. IFN- $\gamma$ - and/or IL-17A-producing CD90.2+CD4+ cells were identified as Ag-specific CD4+ T cells. At Day 7 (top) and Day 30 (bottom) after the last vaccination, gastric Ag-specific CD4+ T cells from these immunized mice were analyzed (C). Absolute number and frequencies of gastric Ag-specific CD4+ $T$ cells among total CD4+ T cells at Day 7 (top) and Day 30 (bottom) were quantified (D). The frequencies of Ag-specific CD4+ T cells from MLN among total CD4+ T cells at Day 7 (top) and Day 30 (bottom) were quantified (E). In all graphs, dots represent individual data points and columns represent median values. ${ }^{\star} P<0.05$, ${ }^{\star * \star} P<0.001,{ }^{\star \star \star \star} P<0.0001, n s=$ not significant. The Kruskal-Wallis test (vs. naïve) was used. Data were pooled from two individual experiments with $n=5$ mice per group.

(i.n.), intramuscular (i.m.) and oral (p.o.) vaccinations, but the number was much lower than that in the GSL control mice (Figures 1C,D). To investigate whether these Ag-specific $\mathrm{CD} 4+\mathrm{T}$ cells could form a gastric memory $\mathrm{T}$ cell pool, we compared the number of gastric Ag-specific CD4+ T cells among different groups on Day 30. Results revealed that few Ag-specific CD4+ $\mathrm{T}$ cells were detected in these groups except for GSL (Figures 1C,D). Moreover, the expansion of Ag-specific CD4+ $\mathrm{T}$ cells was observed on Day 7 in the MLN from mice receiving i.m. or s.c. administration (Figure 1E). These findings suggest that conventional vaccine strategies can drive some of Ag-specific CD4+ T cells presenting in stomach, but these cells fail to give rise to a formidable memory $\mathrm{T}$ cell pool.

\section{Gastric Subserosa Layer Vaccination Recruits Abundant Ag-specific CD4+ T Cells Into Stomach}

Development of an in situ vaccine strategy might be of utmost importance to establish a strong CD4+ Trm pool in stomach $(24,25)$. GSL injection has been used for local anesthesia and for the development of gastric ulcer or in situ tumor animal models. Given that orientation of visible blood vessels is from lesser curvature to greater curvature, we hypothesized that the non-vascular zone of greater curvature was a feasible region to establish a local Ag depot. To verify this, we formulated CCF with Alum adjuvant, performed the laparotomy to access stomach, and injected $5 \mu \mathrm{l}$ vaccine into GSL (Figures 2A,B). Acute 


\section{A \immunization \sacrifice $\downarrow G S L$}
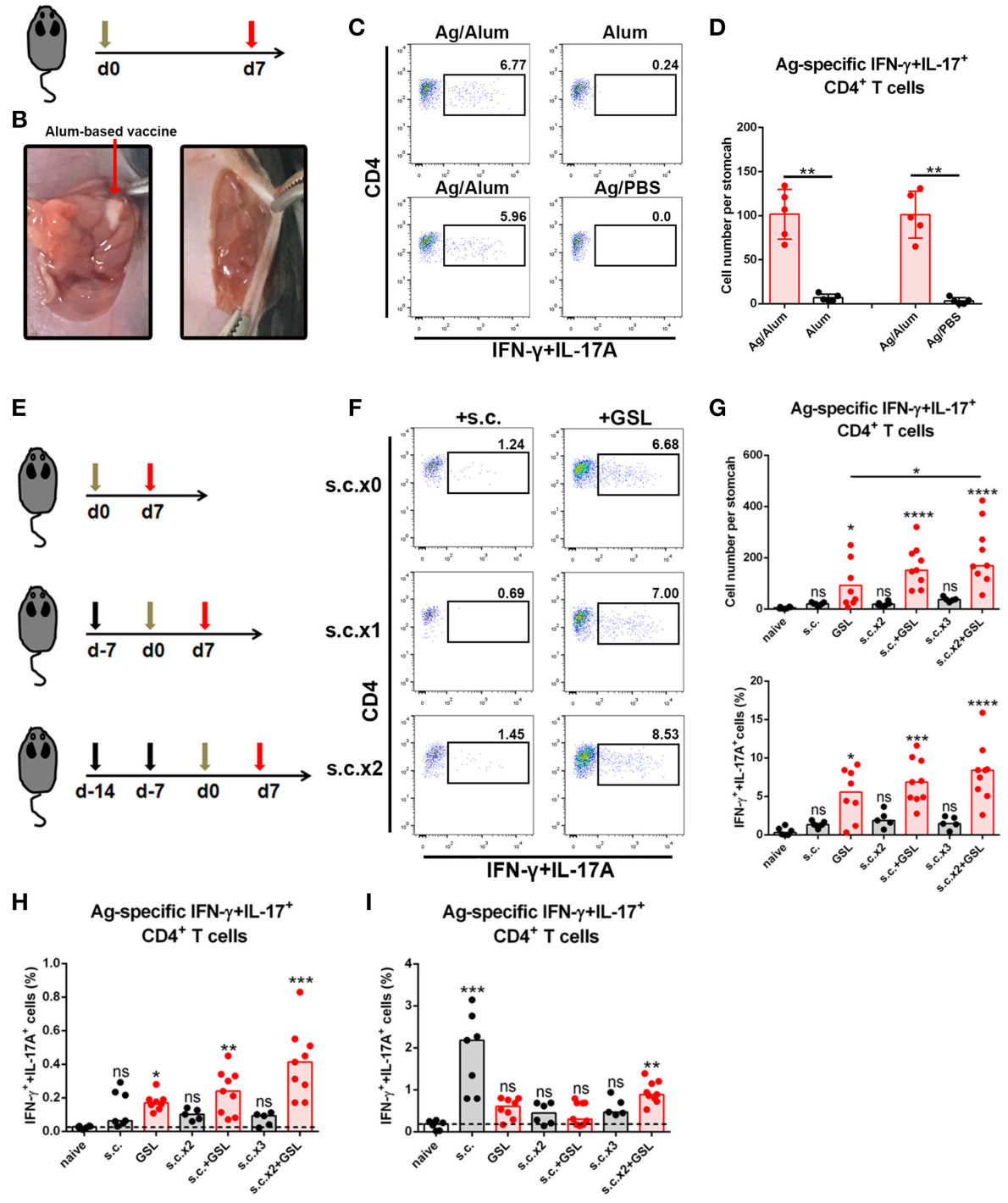

FIGURE 2 | A vaccine strategy triggered abundant Ag-specific CD4+ T cells infiltration of the stomach. (A) C57BL/6J mice were immunized at Day 0 by GSL and sacrificed on Day 7. (B) Details for GSL vaccination are as follows: an incision was made above the stomach and $5 \mu$ l vaccine formulation (containing $\sim 7.5 \mu \mathrm{g}$ ) was injected into the subserosa layer of the stomach. The incisions in peritoneum and skin were sutured. (C) Gastric Ag-specific CD4+ T cells were analyzed in mice that GSL vaccinated with Ag/Alum, Alum and Ag/PBS. (D) Absolute number of gastric Ag-specific CD4+ T cells at Day 7 were quantified (E) C57BL/6J mice were immunized with one of six different strategies. (F) Gastric Ag-specific CD4+ T cells in each group were analyzed as described before. Absolute number and frequencies of gastric Ag-specific CD4+ T cells among total CD4+ T cells at Day 7 were quantified (G). The frequencies of Ag-specific CD4+ T cells from MLN (H) and spleen (I) among total CD4+ T cells were quantified. In all graphs, dots represent individual data points and columns represent median values. ${ }^{\star} P<0.05$, ${ }^{\star \star} P<0.01,{ }^{\star \star \star} P<0.001,{ }^{\star \star \star \star} P<0.0001, n s=$ not significant. The Kruskal-Wallis test (vs. naïve) or Mann-Whitney $U$ test (for two groups) was used. Data were pooled from six individual experiments with $n=5-9$ mice per group.

inflammation, which was characterized by mucosal swelling, was observed in the vaccination site, and abundant Ag-specific CD4+ $\mathrm{T}$ cells could be detected 5-7 days later. To clarify the requirement for Ag-specific CD4+ cell recruitment in GSL vaccination, mice were injected with Ag/Alum, Alum alone, or $\mathrm{Ag} / \mathrm{PBS}$ solution, respectively. Injection of Alum alone failed to recruit any Ag-specific CD4+ T cells, but induced regional tissue swelling (Figures 2C,D). In comparison, GSL injection of
Ag/PBS solution did not recruit Ag-specific CD4+ T cells into stomach either, and no tissue alteration was observed in the injection site. We found that Ag/PBS solution could be absorbed within hours after injection, indicating that a sustained-release vehicle (i.e., Alum adjuvant) was necessary for Ag-specific T cell recruitment.

In consideration of the low dosage of vaccine used in GSL vaccination, we modified the vaccine strategy with an additional 
step to create the following protocol: i) subcutaneous vaccination to induce a systemic Ag response, followed by ii) a surgical operation to inject a micro-dose of vaccine into GSL. To test this strategy for the induction of an Ag-specific T cell response, we performed six kinds of vaccination programs that included multiple rounds of subcutaneous vaccination and/or a GSL vaccination (Figure 2E). As expected, some of gastric Ag-specific $\mathrm{CD} 4+\mathrm{T}$ cells were detected in mice receiving subcutaneous vaccination alone, but abundant these cells were observed in GSL-vaccinated mice. Performing two rounds of subcutaneous vaccination significantly boosted the number of Ag-specific CD4+ T cells recruited by GSL vaccination (Figures 2F,G). We also examined the percentages of Ag-specific CD4+ T cells in spleen and MLN. s.c. vaccination did not induce plenty of Agspecific CD4+ T cells in MLN, whereas Ag-specific CD4+ T cells were abundant in mice receiving s.c. vaccination plus GSL vaccination (Figure $\mathbf{2 H}$ ). In the different vaccination programs, the levels of splenic Ag-specific CD4+ T cells were variable (Figure 2I). We also tested whether GSL injection of mice with Alum or Ag/PBS solution after s.c. vaccination could induce plenty of Ag-specific CD4 $+\mathrm{T}$ cells into stomach. The results showed that without regional Ag exposure and adjuvantinduced inflammation, recruitment of Ag-specific CD4+ T cells was limited (Supplementary Figure 2). These results indicated that introduction of a systemic Ag-specific response enhanced the regional Ag-specific CD4 $+\mathrm{T}$ cell recruitment induced by GSL vaccination.

Taken together, these data indicate that the local Ag encounter, Ag vehicle phase and systemic immune response all contribute to the maximum recruitment of Ag-specific CD4+ T cells induced by GSL vaccination.

\section{Ag-specific CD4+ T Cells Retain in Stomach Long-Term Without Recirculation} To investigate whether a durable Ag-specific CD4+ Trm pool was formed after s.c.x2 +GSL vaccination, we counted the number of Ag-specific CD4+ $\mathrm{T}$ cells in stomach over the following 8 months (Figure 3A). Age-matched naïve mice were served as negative controls to exclude the influence of age. In immune mice, the granulation tissue induced by the Alum adjuvant was stiffened at Day 30 and shrank in the following months (Figure 3B). No gland atrophy or metaplasia was observed in the mucosa near the vaccination site during Days $30-$ 60 (Supplementary Figure 3). On the other hand, contraction of the infiltrating Ag-specific CD4+ T cell population was complete within 30 days and a stable Ag-specific CD4+ memory $\mathrm{T}$ population could be detected for at least 240 days (Figures 3C,D).

In the next step, to investigate whether these CD4+ memory $\mathrm{T}$ cells experienced recirculation via the lymphovascular system, we treated immune mice with FTY720 daily to inhibit lymphocyte egress from lymph nodes for 14 days (Figure 4A). Even though circulating CD4+ T cells were decreased by more than 100-fold in blood and Ag-specific CD4+ memory T cells vanished from blood, the number of Ag-specific CD4+ memory $\mathrm{T}$ cells was stable in stomach (Figures 4B,C), suggesting a characteristic of local retention. Moreover, the absolute number of Ag-specific
CD4+ memory T cells was not altered in MLN, but significantly decreased in spleen after FTY720 administration (Figures 4B,C).

To verify whether these Ag-specific CD4+ Trm cells were sensitive to systemic CD4 antibody depletion, we i.p. injected immune mice with anti-CD4 antibody (Figure 4D). No CD4+ $\mathrm{T}$ cells could be detected in blood or spleen, whereas Ag-specific $\mathrm{CD} 4+$ cells in stomach remained numerically unchanged and measurable, although with lower CD4 expression (Figures 4E,F), implying their retention in a distinct anatomical location which was less affected by circulation. Collectively, these data reveal that infiltrating Ag-specific CD4+ Teff cells can form a longlived Trm pool and most of these cells may be separated from circulation.

\section{Distribution of These CD69+CD103-CD4+ Trm Cells Is Dependent on Epithelial Architecture of Stomach}

We next examined the location of $\mathrm{CD} 4+\mathrm{T}$ cells induced by GSL vaccination. Stomach was isolated and cut through lesser curvature. As shown in Figure 5A, vaccination region was sniped longitudinally and used for immunohistological staining of CD4+ T cells. Six groups with different vaccination programs were involved in this experiment. Mice were sacrificed on 7day or 30-day post GSL vaccination. The results indicated that abundant CD4 $+\mathrm{T}$ cells could be observed in the gastric mucosa near the vaccination site at 7 -day post GSL vaccination (Figures 5B,D). Infiltration of CD4+ T cells was restricted to the adjacent mucosa, as few CD4+ cells presented in the mucosa of non-vaccination site. Density of CD4 $+\mathrm{T}$ cells was not associated with Ag exposure and prime. Extremely low density of CD4+ $\mathrm{T}$ cells was observed in stomach of naïve and s.c. immunized mice in the same perspective. Additionally, only some CD4+ $\mathrm{T}$ cells appeared around the vaccination site, even though the surrounding region was enriched with immune cells (Figure 5D). At 30-day post GSL vaccination, density of CD4+ $\mathrm{T}$ cells on mucosa of vaccination site was decreased (Figures $5 \mathrm{C}, \mathrm{E}$ ). Notably, at this time point, most CD4+ T cells were located close to the epithelium, which was confirmed by the distinct epithelial architecture of stomach (Figures 5E,F). For instance, in the body mucosa close to the cardia equivalent, CD4+ Trm cells formed a chain; in the middle of the body mucosa, CD4+ Trm cells were evenly distributed in the epithelial region of gastric pit; and in the transition region, both two retention patterns could be observed. Moreover, $10 \mu \mathrm{m}$ sections of gastric tissue indicated that these CD4+ T cells were in contacted with the epithelial cells (Figure 5E, bottom). Parallelly, detection of Ag-specific CD4+ $\mathrm{T}$ cells in the epithelium or lamina propria also suggested that at memory stage, most of Ag-specific CD4+ cells were located on epithelial regions (Figures $\mathbf{5 G}, \mathbf{H}$ ). These data demonstrate that CD4+ T cells recruited by GSL vaccination preferentially infiltrate the adjacent mucosa and survive in the special niches of the epithelium.

Previous studies suggest that CD4+ Trm cells are maintained in vagina, intestine and skin, and cluster with macrophages, dendritic cells (DCs) and CD8+ T cells (24, 26, 27). To examine whether other immune cells were responsible for CD4+ Trm 


\section{A} \immunization \sacrifice \GSL

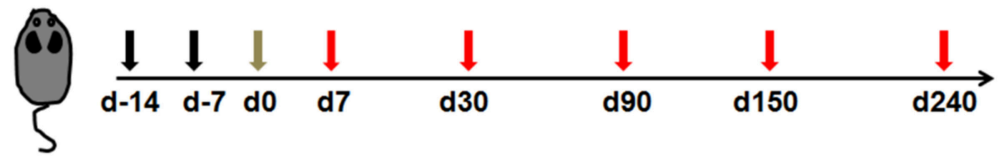

B

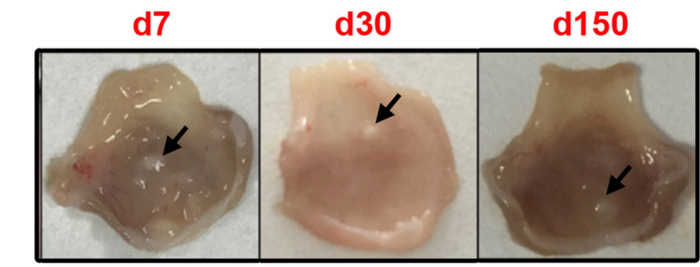

C
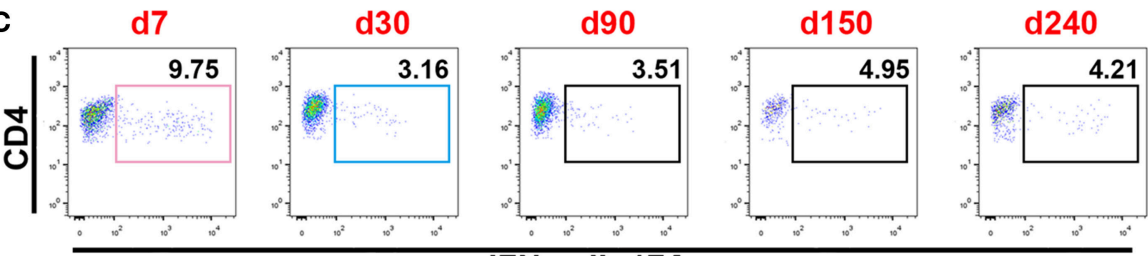

IFN- $\gamma+$ IL-17A
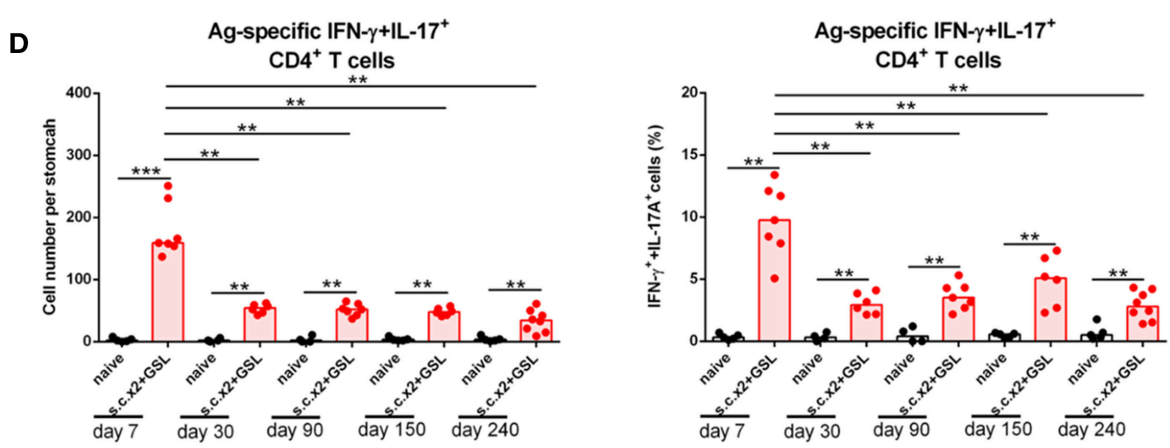

FIGURE 3 | Ag-specific CD4+ Teff cells contracted and sustained in stomach long-term. (A) C57BL/6J mice were s.c. immunized with Ag/Alum at Days-14 and-7, and treated with GSL vaccination at Day 0. (B) Mice were sacrificed on Day 7, Day 30, and Day 150, and their stomachs were unfolded. Arrows indicated granulation tissue in the vaccination sites. (C) Mice were sacrificed on the day indicated in the timetable (red arrows), and the gastric Ag-specific CD4+ T cells in each time point were analyzed as described previously. (D) Absolute number and frequencies of gastric Ag-specific CD4+ T cells among CD4+ T cells at each time points were quantified. In all graphs, dots represent individual data points and columns represent median values. ${ }^{\star \star} P<0.01,{ }^{\star \star \star} P<0.001$. Mann-Whitney $U$ test was used to compare two groups. Data were pooled from six individual experiments with $n=4-8$ mice per group.

cell residence in stomach, we analyzed the types of infiltrated immune cells at whole organ level on Day 7 and Day 30 (Figure 6A). All cell types except macrophages, i.e., neutrophils, inflammatory monocytes, mo-DCs, B cells, $\gamma \delta \mathrm{T}$ cells, CD4+ T cells and CD8+ T cells, were expanded on Day 7 (Figure 6B). However, flow cytometric analysis showed that most of the immune cells seceded from stomach before Day 30, suggesting that the increased gastric immune cell content induced by GSL vaccination was not sustainable at the whole organ level. To visualize the regional relationship between CD4 $+\mathrm{T}$ cells and innate immune cells, an immunolocalization assay was performed and the images indicated no direct relationship between the CD4+ and CD11b + cells during the memory stage, as the $\mathrm{CD} 11 \mathrm{~b}+$ cells preferentially enveloped the vaccination site (Figure 6C). Next, we measured several chemokines CCL5,
CXCL9 and CXCL10 that are critical for CD4+ T cell recruitment (26). Compared with memory stage, CCL5 and CXCL10 levels were significantly increased in the vaccination site during effector stage (Figure 6D). Interestingly, at memory stage, CCL5, CXCL10, and TGF- $\beta 1$ levels at the vaccination site were lower than those of naive mice (Figure 6E). We reckoned that the granuloma structure might affect regional homeostasis. In total, these data demonstrate that a distinct migration and retention pattern of CD4+ T cells is induced by GSL vaccination.

Next, we isolated intraepithelial T cells from the vaccination site and analyzed their phenotypes at the memory stage. CD4+ $\mathrm{T}$ cells from the vaccination site expressed CD69 and CD44, but expressed little CD103 and no CD62L; on the contrary, CD8+ $\mathrm{T}$ cells in this region expressed CD103, CD69, and CD44 (Figure 6F). Parallelly, we measured the expression of 

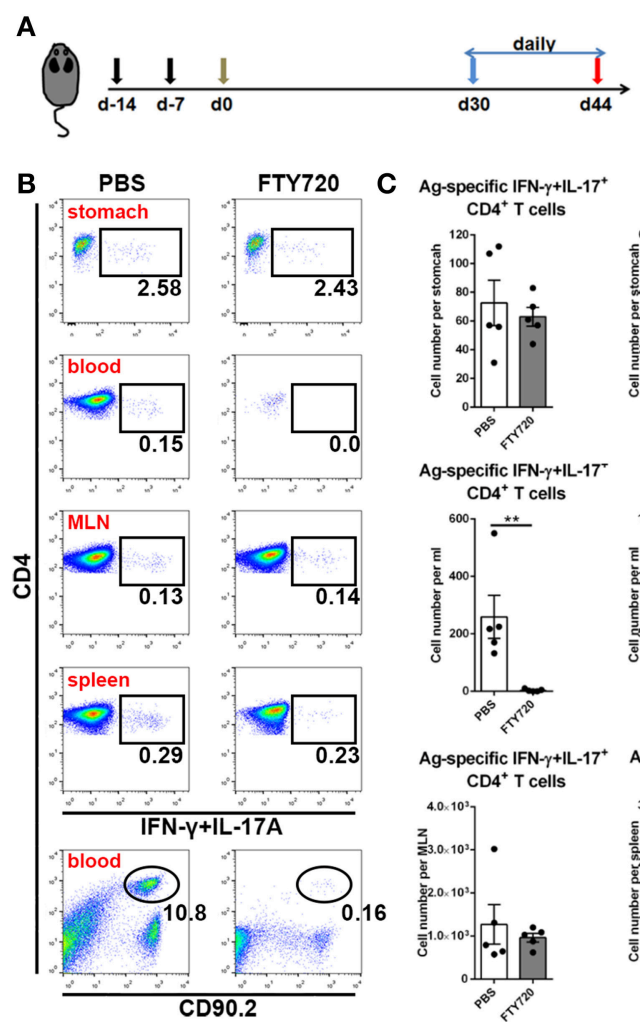

Ag-specific IFN- $-\mathrm{IL}-17^{+}$
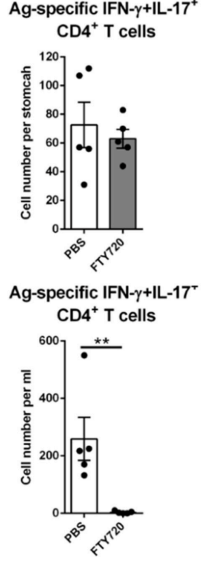

Ag-specific IFN- $\gamma^{+1 \mathrm{LL}-17^{+}}$

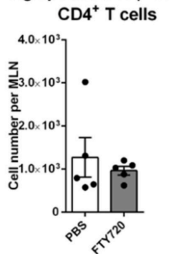

D

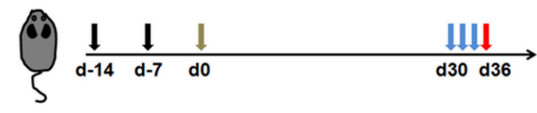

E
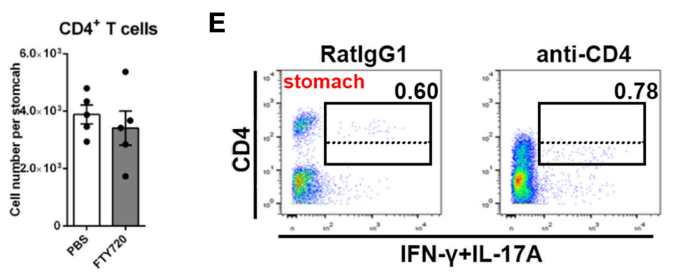

Ag-specific IFN- $\gamma+$ IL-17
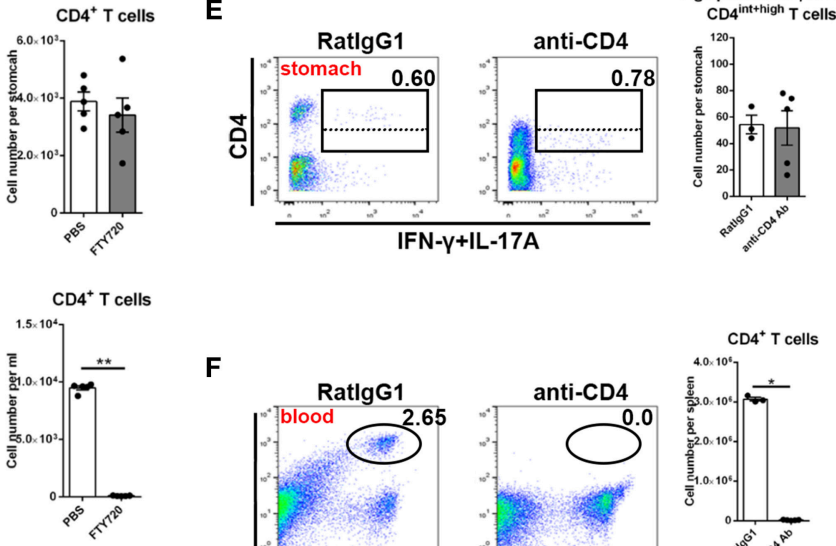

Ag-specific IFN- $\gamma+\mathrm{IL}-17^{+}$

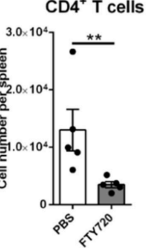

$\mathbf{F}$

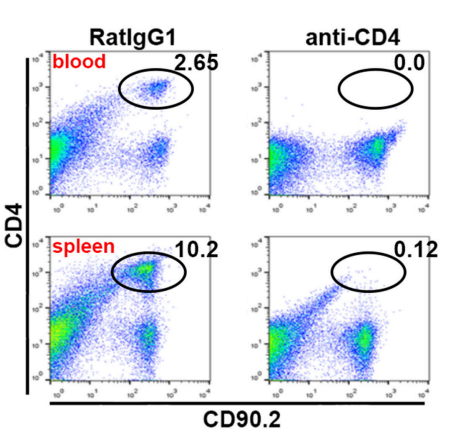

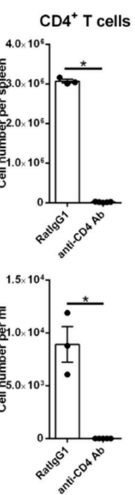

FIGURE 4 | Gastric Ag-specific CD4+ memory T cells induced by GSL vaccination experienced little recirculation and were located separately from the blood. (A) C57BL/6J mice were s.c. immunized with Ag/Alum at Days-14 and-7, and treated with GSL vaccination at Day 0. Thirty days later, the mice were i.p. injected with 1 $\mathrm{mg} / \mathrm{ml}$ of FTY720 or PBS daily for 14 days. (B) Ag-specific CD4+ T cells among CD4+ T cells in the stomach, blood, MLN and spleen and CD4+ T cells among total leukocytes were analyzed. (C) Absolute number of Ag-specific CD4+ T cells in stomach, MLN, blood and spleen and CD4+ T cells in stomach and blood at the whole organ level were quantified. (D) 30-day post GSL vaccination, mice were i.p. injected with $100 \mu \mathrm{g}$ anti-CD4 antibody or anti-RatlgG1 antibody at Days 30, 32 and 34. Mice were sacrificed on Day 36. (E) Ag-specific CD4+ T cells among total CD90.2+ cells in stomach were shown (left), and the absolute number of Ag-specific CD4+ T cells in stomach was quantified (right). (F) CD4+ T cells among total leukocytes in blood or spleen were shown (left), and the absolute number of CD4+ T cells in blood or spleen was quantified (right). In all graphs, dots represent individual data points and columns represent mean and SEM. ${ }^{*} P<0.05$, ${ }^{* *} P<0.01$. Mann-Whitney $U$ test was used to compare two groups. Data were pooled from two individual experiments with $n=3-5$ mice per group.

CD69 and CD103 on Ag-specific CD4+ Trm cells. Almost all these cells displayed a CD69+CD103-phenotype (Figure 6G). These data indicate that GSL vaccination induces intraepithelial CD69+CD103-CD4+ Trm cells in stomach.

\section{Vaccine Strategy Involving CD4+ Trm Cells Provides Rapid and Long-Term Protection Against Helicobacter Insult}

Vaccination programs used in previous studies generally performed $H$. pylori challenge on $\sim 14$ days after the last vaccination $(28,29)$. At this time point, Teff cells induced by conventional vaccinations might not completely attenuate in blood or stomach of immune mice. A previous clinical study indicated that the protective effects induced by oral vaccination continued to attenuate in the years that followed (30). Here, we tested the differences between conventional vaccinations and vaccine strategies involving GSL vaccination in terms of rapid and long-term protection. Mice were divided into nine groups and subjected to different immunization programs (Figure 7A). H. pylori challenge was performed 60 days later. At Day 63, colonization of $H$. pylori was determined by quantitative culture. Substantial reductions of bacterial colonization could be found in mice that received GSL vaccination, and s.c.x $2+$ GSL vaccination provided the optimal protection at this time point (Figure 7B), indicating that these mice demonstrated rapid antimicrobial responses and prolonged protection. In comparison, most conventional vaccine strategies failed to reduce bacterial colonization and no reduction of $H$. pylori colonization was observed in mice receiving Alum alone by GSL vaccination. Furthermore, we investigated the limit of anti-microbial response involving CD4+ Trm cells (Figure 7C). Results showed that s.c.x2+GSL vaccination induced a drastic reduction of $H$. pylori load at day $0-14$ and the bacterial load was sustained at a low degree during day 14-30 post challenge (Figure 7D). A numerical advantage of reduction was observed between s.c.x2+GSL vaccination and GSL vaccination. These results imply that s.c.x2+GSL vaccination induces rapid and long-term protection against H. pylori. 

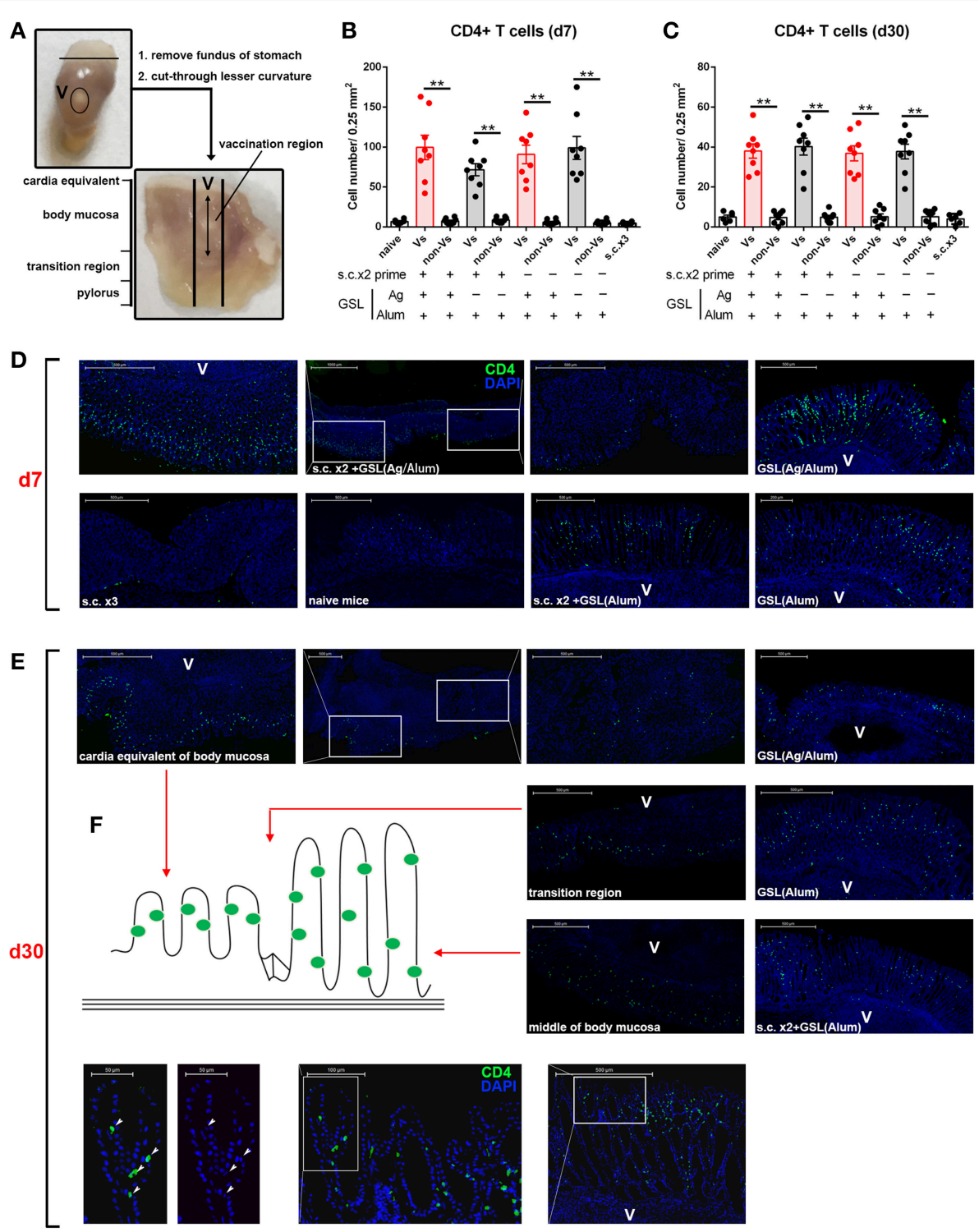

G

EL

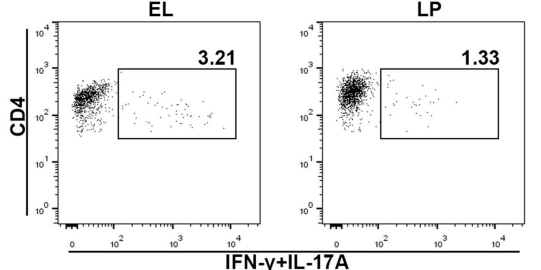

H

Ag-specific IFN- $\mathrm{\gamma}^{+\mathrm{IL}-17^{+}}$

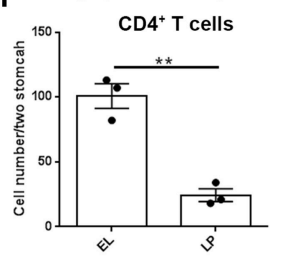

FIGURE 5 | GSL vaccination drove CD4+ T cell infiltration of the mucosa and retention within the epithelium. (A) Stomach was dissected as photo indicated and anatomical position was shown in the macroscopic perspective. Gastric tissue from mice that were treated with GSL vaccination was cut longitudinally and stained with Alexa Fluor ${ }^{\circledR}$ 488-anti-CD4 antibody. CD4+ T cells were quantified on immunofluorescent staining of $20 \mu \mathrm{m}$ frozen sections from Day 7 (B) or Day 30 (C) post GSL vaccination. (D,E) Representative images of immunofluorescent staining (green, CD4; blue, DAPI; V, vaccination site). (F) Schematic plan of CD4+ cell location (left) and the distribution of CD4+ cell on gastric middle mucosa and transition region (right). The precise positions of CD4+ cells were determined by immunofluorescent staining of $10 \mu \mathrm{m}$ frozen sections (green, CD4; blue, DAPI; V, vaccination site). ${ }^{\star \star} P<0.01$. Mann-Whitney $\mathrm{U}$ test was used to compare two groups. Data were repeated 3-8 times. (G) EL or lamina propria (LP) lymphocytes were pooled from two immune mice at memory stage. Ag-specific CD4+ T cells were quantified $\mathbf{( H )}$. Six mice $(n=6)$ were used in this experiment. ${ }^{\star \star} P<0.01$, unpaired $t$-test. 
A

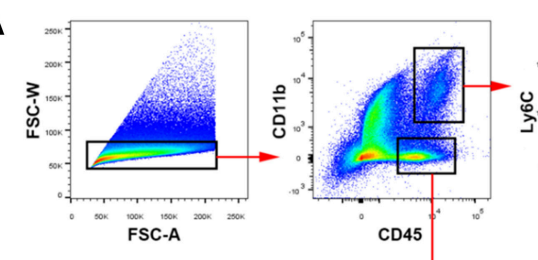

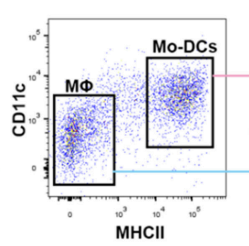

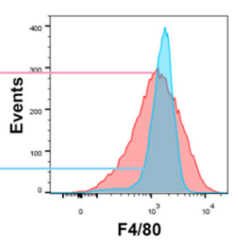

GR-1
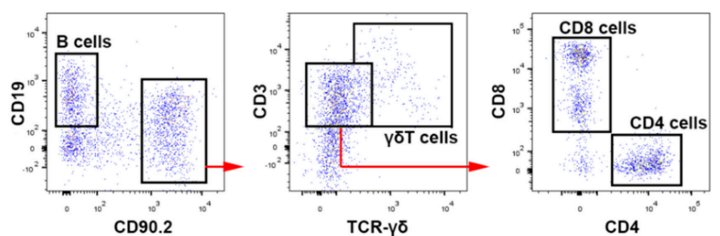

C
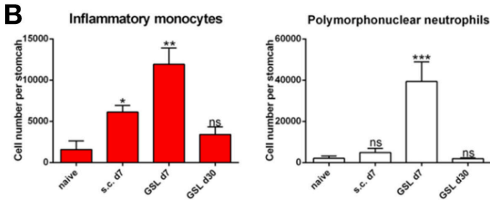

B cells

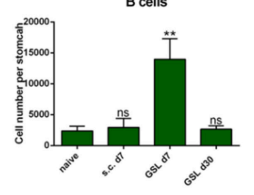

$\gamma \delta \mathrm{T}$ cell

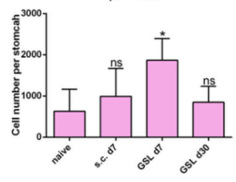

Macrophages

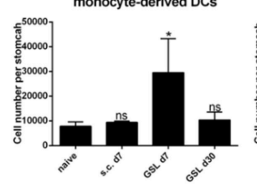

CD4 T cells
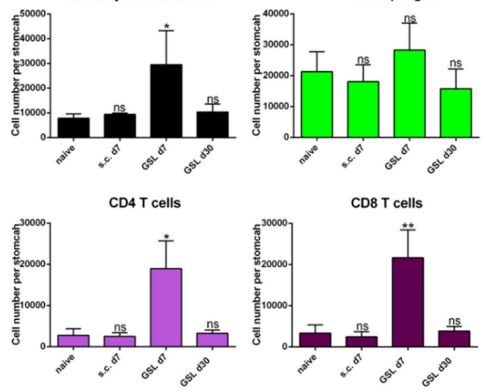

CD8 T cells

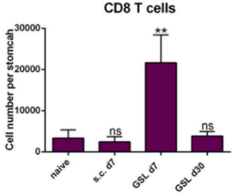

F Gate on SSC low CD45+CD90.2+
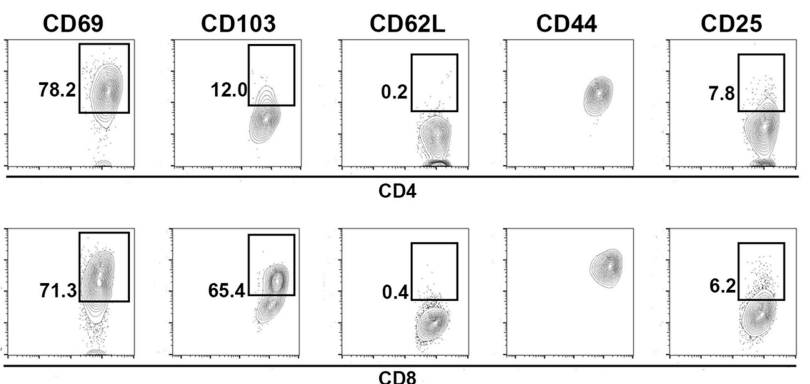

D

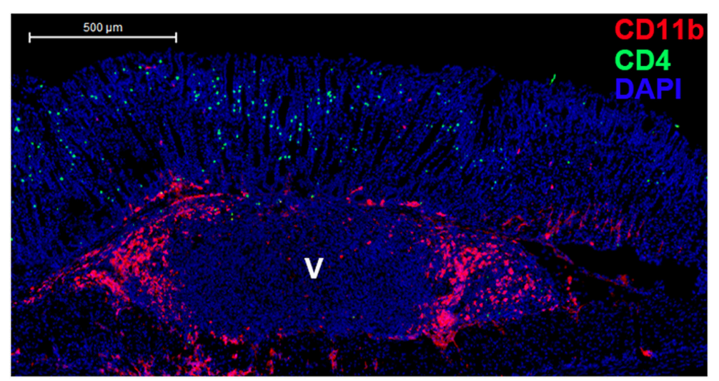

GSL-Nonvs (d 30)

$\Delta$ GSL-Vs (d 2)

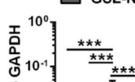
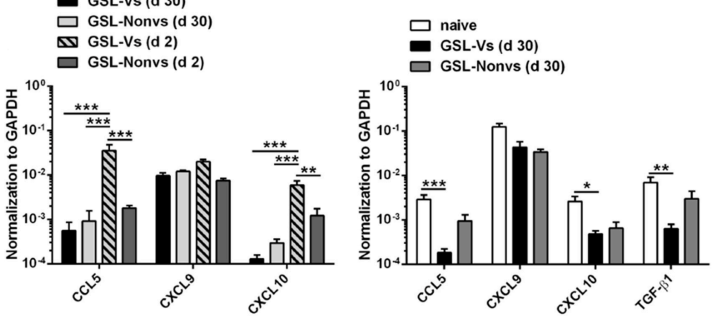

G

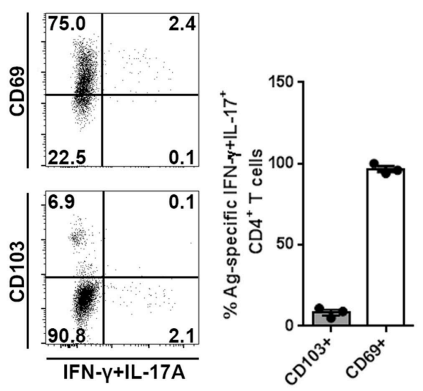

FIGURE 6 | The maintenance and phenotype of CD4+ Trm cells. (A) GSL vaccination was performed at Day 0 and mice were sacrificed on Day 7 and Day 30 to analyze immune cell infiltration using the indicated gated strategy. (B) Absolute number of innate and adaptive immune cells at the whole organ level was quantified. ${ }^{\star} P<0.05$, ${ }^{\star \star} P<0.01$. The Kruskal - Wallis test (vs. naïve) was used. Columns represent mean and SD. Data were pooled from two individual experiments with $n=5-6$ mice per group. (C) Immunofluorescent staining of $20 \mu \mathrm{m}$ frozen sections at Day 30 (green, CD4/CD11b; red, CD11b; blue, DAPI; V, vaccination site) post GSL vaccination. Data were repeated at least three times with similar results. (D) CCL5, CXCL9, and CXCL10 mRNA were measured at Day 2 and Day 30 by qRT-PCR. GSL-Vs: vaccination site; GSL-Non vs: non-vaccination site. (E) CCL5, CXCL9, CXCL10 and TGF- $\beta 1$ mRNA were measured at Day 30 by qRT-PCR. (F) Phenotypes of intraepithelial CD4+ T cells and CD8+ T cells in the vaccination region. (G) Expression of CD69 and CD103 was analyzed on Ag-specific CD4+ Trm cells. ${ }^{\star} P<0.05,{ }^{\star \star} P<0.01,{ }^{\star \star \star} P<0.001$. Mann-Whitney $\cup$ test was used to compare two groups. Data were pooled from two individual experiments with $n=3-5$ mice per group. Columns represent mean and SEM. 


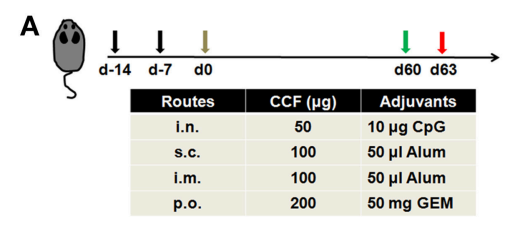

B

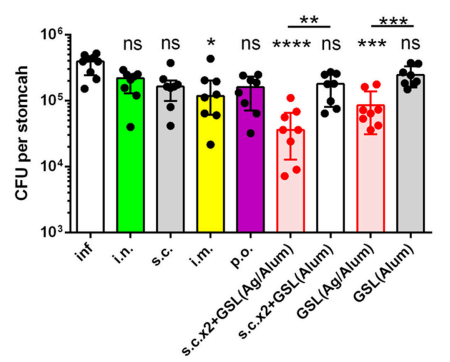

C

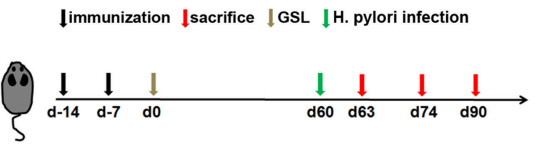

D

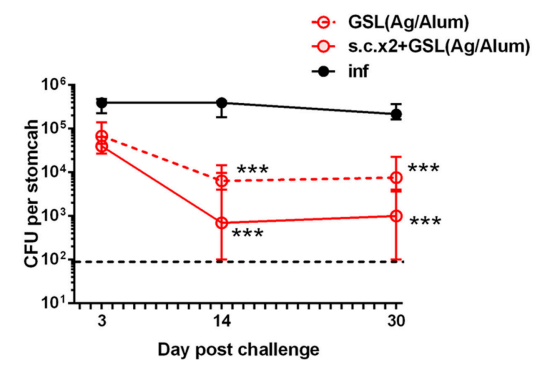

FIGURE 7 | s.cx2+GSL vaccination provided prolonged and rapid protection against Helicobacter insult. (A) C57BL/6J mice were immunized at Day-14, Day-7, and Day 0 with different vaccine formulation described in the table. Helicobacter challenge was performed on Day 60 and mice were sacrificed on Day 63 to test the protective effects. (B) $H$. pylori colonization was determined by the quantitative culture on Day 63 . ${ }^{\star} P<0.05,{ }^{\star * \star} P<0.001,{ }^{\star * \star \star} P<0.0001, n s, P>0.05$. The Kruskal-Wallis test (vs. naïve) was used. Data were pooled from two individual experiments with $n=8$ mice per group. (C) Mice experienced s.cx2+GSL vaccination were challenged at Day 60 and $\mathrm{H}$. pylori colonization was determined on Day 63, Day 74 , and Day 90 (D). ${ }^{\star \star} P<0.05$, ${ }^{\star \star \star} P<0.01$. Mann-Whitney $U$ test was used to compare two groups. In all graphs, columns represent median and interquartile. Data were pooled from two individual experiments with $n=6-8$ mice per group.

\section{Reactivated CD4+ Trm Cells Trigger a Rapid Systemic Th1/Th17 Cellular Response to Support Tissue-Wide Anti-microbial Response}

Next, we investigated the immunological mechanism of protective response and the role of circulating lymphocytes in mice receiving s.c.x $2+$ GSL vaccination. Administration of FTY720 to immune mice impaired the protective effects, and depletion of CD4+ T cells by an anti-CD4 antibody completely abrogated protection (Figure 8A), indicating the vital roles of circulating memory lymphocytes and CD4+ cells in protective response induced by GSL vaccination. Furthermore, IFN- $\gamma$ and IL-17A depletion by neutralizing antibody partly suppressed the protective response in immune mice, highlighting the roles of IFN- $\gamma$ and IL-17A in providing protection (Figure 8C). Analysis of Ag-specific immune response at day 3 post challenge suggested that a potent Ag-specific Th1/Th17 cell response in stomach and the expansion of Ag-specific Th17 cells in spleen already could be detected (Supplementary Figure 4A). Next, we compared the Ag-specific Th1/Th17 cell responses in immune mice treated with or without FTY720 in stomach, blood, spleen, and MLN at 7-day post challenge. FTY720 administration significantly dampened the expansion of Ag-specific Th1 and Th17 cells in stomach and blood (Figures 8D-F). In addition, after preventing lymphocyte egress from the lymph nodes with FTY720, the percentages of Ag-specific Th1 cells in spleen and MLN were increased by 3-fold; however, an increase in the percentage of Ag-specific Th17 cells was only observed in MLN (Figures 8D-F). Because of the powerful ability of Th17 cells to induce an anti-microbial response, reactivated MLN-settled Ag-specific memory Th17 cells might be a vital source of gastric Th17 cells that contribute to the recruitment of innate inflammatory cells and trigger tissue-wide protection. In fact, at 7-day post challenge, the mucosa near the vaccination site showed fewer inflammatory cell infiltration as compared with the distal mucosa (Supplementary Figure 4B), possibly suggesting a tissue-wide inflammatory cell infiltration was temporally delayed to the regional infiltration. These data highlight the alarming function of these intraepithelial CD4+ Trm cells, and indicate that regionally positioned CD4+ Trm cells can trigger tissue-wide $H$. pylori clearance through the recruitment of circulating Th1/Th17 cells.

\section{DISCUSSION}

Stomach is an inhospitable digestive organ that is inhabited by only $\sim 200$ different species (8). The notorious one is H. pylori, which is a helical rod-shaped organism that is in contact with the gastric epithelium and influences the physiology of gastric stem cell pool by inducing chronic inflammation (31). Evidence indicates that as long as 50,000 years of coevolution with human have conferred multiple capabilities (e. g., secretion of virulence factors and remodeling of autologous constituents) on $H$. pylori to adapt to the milieu of stomach and escape the host defensive mechanisms (32-35). Likewise, these capabilities lead to an undesirable outcome for $H$. pylori vaccines. Conventional vaccine strategies have been extensively tested in the field of $H$. pylori vaccinology $(15,16,36-38)$, while no study identified an Ag-specific cellular response in stomach. Here, we demonstrated that by using intracellular cytokine staining, a small population of Ag-specific CD4+ T cells could be measured during effector stage rather than memory stage of these immunizations, suggesting conventional vaccine strategies were less effective to induce a measurable CD4+ Trm pool 
A \immunization \sacrifice \GSL \H. pylori infection \FTY720 or neutralizing Ab

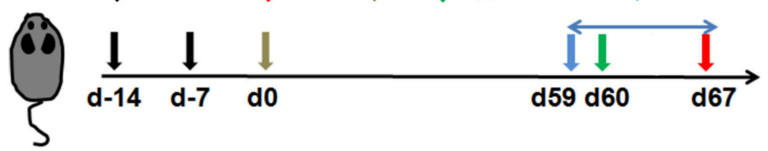

B

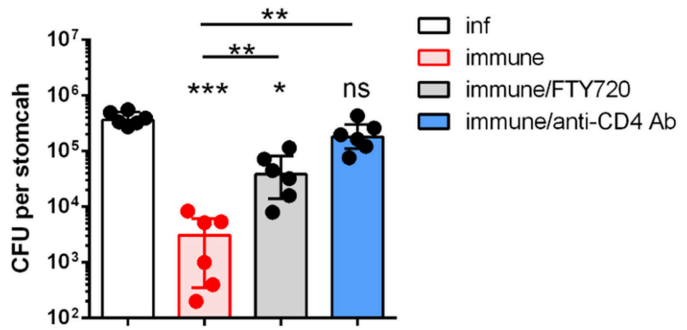

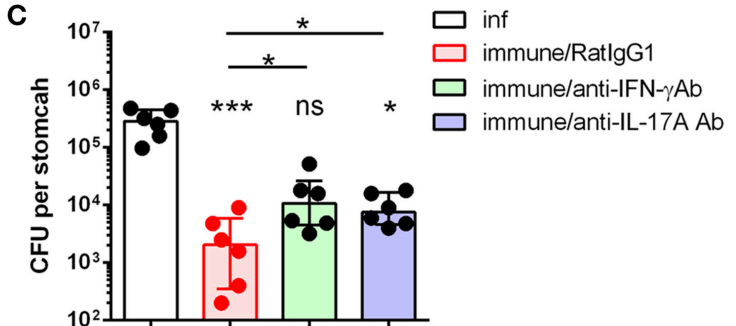

D

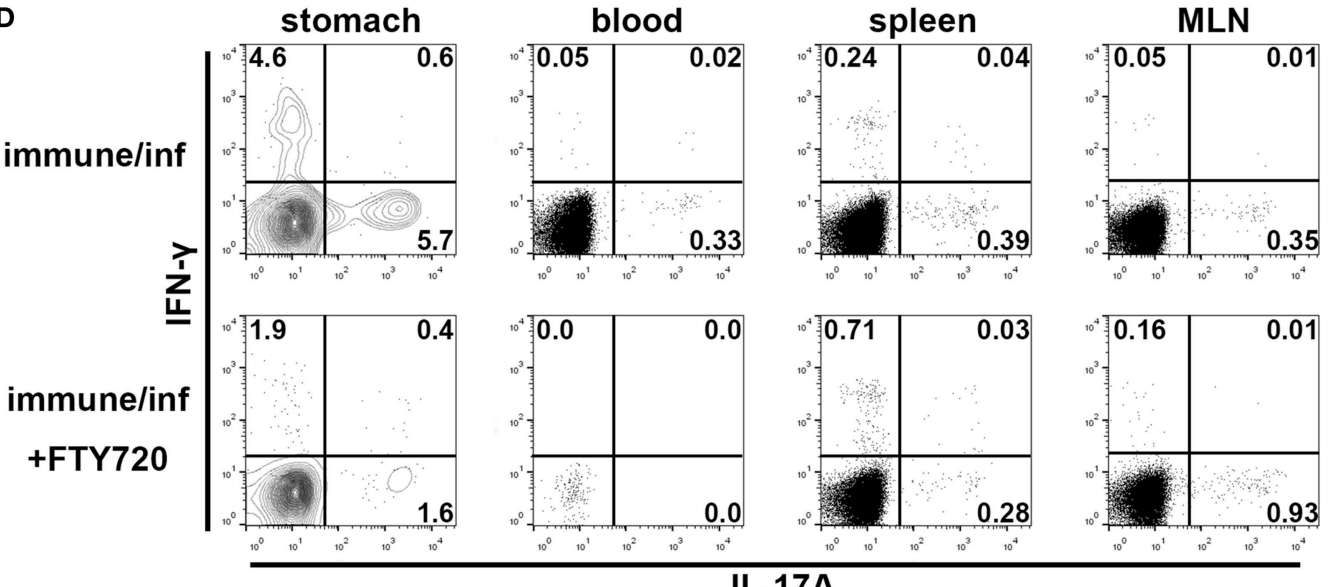

E

$$
\text { Ag-specific IFN- } \gamma^{+} \mathrm{CD}^{+} \text {T cells }
$$

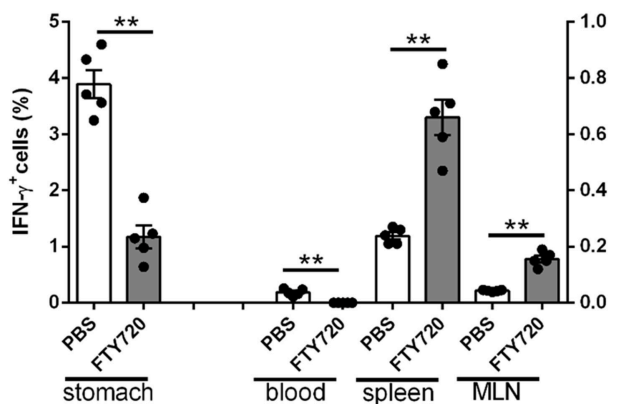

$\mathbf{F}$

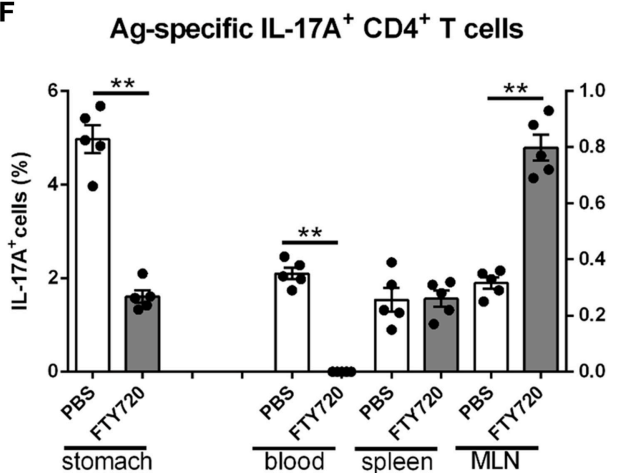

FIGURE 8 | The immunological mechanism of protective response in the mice experienced s.c.x2+GSL vaccination. (A) Mice experienced s.cx2+GSL vaccination. H. pylori challenge was performed on Day 60. FTY720 administration (1 mg/kg) was performed daily during Days 59-67. Neutralizing antibody (i.p.) injection was performed on Days 59, 61, 63, and 65. H. pylori colonization were determined on Day 67 after FTY720 and anti-CD4 antibody administration (B) or after anti-IFN- $\gamma$ and anti-IL-17A antibodies administration (C). ${ }^{\star} P<0.05,{ }^{\star \star \star} P<0.001, n s=$ not significant. The Kruskal-Wallis test (vs. naïve) was used or The Mann-Whitney U test was used to compare two groups. Dots represent individual data points and columns represent median and interquartile. Data were pooled from two individual experiments with $n=6$ mice per group. (D) Immune mice were administrated with $1 \mathrm{mg} / \mathrm{kg}$ FTY720 daily during Days 59-67 and sacrificed on Day 67 for Ag-specific CD4+ T cell analysis. The percentages of Ag-specific IFN- $\gamma$ (E) or IL-17A (F) CD4+ T cells among total CD4+ T cells in stomach, blood, spleen, and MLN were quantified. ${ }^{\star \star} P<0.01$, The Mann-Whitney $U$ test was used to compare two groups. Dots represent individual data points and columns represent mean and SEM. Data were pooled from two individual experiments with $n=5$ mice per group.

for further investigation. Technological barrier for identifying endogenous Ag-specific CD4+T cells in stomach is obvious. The inefficiency of lymphocyte isolation from stomach of mice has already been proved that $98.3 \%$ lymphocytes loss in stomach using flow cytometry for counting $(2,39)$. A need for purification to ex vivo culture and extensive steps of intracellular cytokine 
staining may lead to the additional cell loss (40). Thus, we decided to establish a mice model with a measurable CD4+ Trm pool.

Past studies have revealed that almost always Trm cells form within the tissue after resolution of inflammation or infection (4, 41). Most $H$. pylori vaccines, including the only licensed $H$. pylori vaccine, are composed of non-infectious $H$. pylori Ags that are poorly immunogenic (30). In consideration of the importance of local inflammation, we employed GSL injection to deliver a micro-dose of Alum-based $H$. pylori vaccine into stomach and subcutaneous vaccination was performed before GSL vaccination to mount the infiltrating Ag-specific CD4+ $\mathrm{T}$ cells. This immunization strategy established a stratified immune memory involving the local long-lived CD4+ Trm cells and adjacent lymph node-settled memory $\mathrm{T}$ cells. Upon GSL vaccination, the regional dissemination of Ag-specific CD4+ $\mathrm{T}$ cells was dependent on local Ag recognition and adjuvantinduced inflammation, as either injection with Ag in PBS or delivering Alum adjuvant alone showed no effect on Ag-specific CD4+ T cell recruitment.

Our results also provided insights of CD4+ Trm cells induced by GSL vaccination in terms of migration properties, location/development, and cell surface phenotype. For migration properties, the magnitude of CD4+ Trm cells was stable after FTY720 administration, suggesting they were accord with the important identification parameter that undergoes little or no recirculation (1). For location/development, we found that in GSL immune mice, CD4+ Trm cells showed a distinct pattern of intraepithelial retention. Three phases of their development can be described as followed: in acute phase, CD4+ T cells infiltrated into the mucosa along with the elevated levels of CCL5 and CXCL10 and expansion of various innate immune cells; during the phase of inflammation resolution, more than $60 \%$ of CD4+T cells, including Ag-specific CD4+T cells, withdrew from stomach; in memory stage, CD4+Trm cells were distributed along with the architecture of gastric epithelium and keep stable in magnitude for long-term. An elegant study indicates that vaginal CD4+ Trm cells induced by an attenuated herpes simplex virus 2 sustain in a unique lymphocyte structure, named memory lymphocyte cluster, which is located in parenchyma tissue (26). Half of skin CD4+ T cells persist in peri-follicular clusters that accurately equilibrate with the blood lymphocytes during steady state, and infection can increase the immune cell content of these clusters (27). Our data showed that no lymphocyte clusters were observed in stomach of immune mice in memory stage. Residential pattern of the CD4+ Trm cells in our study was different from the prevailing view that after resolution of infection/inflammation, CD4+ Trm cells are preferentially localized within parenchymal tissues, while CD8+ Trm cells adhere to epithelial layers (1). Current knowledge about Trm cells is primarily obtained from invasive pathogens, which can disseminate into the host organ. Differences on types of vaccine, tissue architecture and inflammatory signaling may be responsible for the outcome of CD4+ Trm cell location/development $(1,25,42,43)$. In addition, the cell surface phenotypes of Ag-specific CD4+ Trm cells isolated from
GSL immune mice were exclusively CD69+CD103-. A recent study in the context of Candida albicans infection finds that non-recirculating skin CD69+CD4+ Th17 cells are sufficient to trigger sterilizing immunity (44). Also, Ag-specific CD4+ Trm cells reported by N. Iijima and A. Iwasaki's study expressed CD69 but little CD103 (26).

Trm cells within peripheral tissues provide strong protection against pathogenic insult $(26,45,46)$. Inducing a potent mucosal immune memory is favored as $H$. pylori restrictedly survive in epithelium of stomach with less invasiveness. During $H$. pylori insult, evenly distributed intraepithelial CD4+ Trm cells are optimally positioned to eliminate the window period and initiate a protective response immediately. GSL vaccination-induced CD4+ Trm cells are long-lived and pathogen-specific, therefore providing prolonged protection that was highly sensitive to $H$. pylori insult. As Th1 and Th17 immunity was highlighted in the anti-microbial response of $H$. pylori vaccine $(28,29)$, we reported that CD4+ Trm cells induced by GSL vaccination sustained in stomach for long-term and rapidly reactivated to recruit circulating Th1/Th17 cells to clear gastric H. pylori. Tissue-autonomous protection was found in the immune mice, but superior antimicrobial effects were dependent on the engagement of circulating lymphocytes (Figure 8B). It might be that protective CD4+ Trm cells induced by GSL vaccination were restricted to adjacent mucosa at low magnitudes and needed the help of circulating Th1/Th17 cells to trigger tissue-wide protection (41). This observation is consistent with previous study that small numbers of Trm cells trigger an antiviral state through amplifying innate or adaptive immune signals (47).

In the present study, we attempted to introduce a vaccineinduced CD4+ Trm pool in stomach and evaluated its protective efficacy. Alum-based vaccine was used for GSL vaccination, but we found an undesirable granuloma that affected regional homeostasis. Employing a biocompatible vehicle with the characteristics to spread in GSL is interested to improve the outcome of this model. Our further study will use the silk fibroin to replace Alum adjuvant to extend the CD4+ Trm cell distribution, increase their magnitudes, and prevent the form of granuloma. A more attractive but challenging question is how to design a feasible delivered system that targets stomach. The laparotomy used in mice is impractical in humans. Combining endoscopy technology with GSL vaccination is also less feasible for the translational application. Recently, an impressive study reports an ingestible self-orienting system for oral delivery of macromolecules that deliveries insulin through gastric mucosa (48). This delivered system is applicable for stomach-targeted vaccination after some adjustment.

Overall, our study developed a mice model with a strategic CD4+ Trm pool in stomach. CD4 $+\mathrm{T}$ cells induced by GSL vaccination preferentially infiltrated the adjacent mucosa, and then restrictively sustained in the epithelial region adjacent to the vaccination site. The underlying mechanism of local maintenance is currently unknown but may be associated with the metabolism of free fatty acids and TGF- $\beta$ signals, similar to the maintenance requirements for intraepithelial 
CD8+ Trm cells (49). Principally, our results indicate that pathogen-specific CD4+ Trm cells within the gastric epithelium can catch the best chance to sound the alarm, orchestrate the defense response, and provide prolonged protection. The notion that developing vaccine strategies involves a Trm population may shed new light on the development of H. pylori vaccines.

\section{ETHICS STATEMENT}

All animal experiments were approved by the Animal Ethical and Experimental Committee of China Pharmaceutical University.

\section{AUTHOR CONTRIBUTIONS}

WL and YX designed all experiments. WL performed surgical operation. ZZ, SL, CH, NX, and $\mathrm{AH}$ conducted the animals. WL, ZZ, and SL analyzed the data. ZZ prepared the reagents and the experiments. WL, YX, LZ, ES, and TX discussed the results and wrote the manuscript. All authors have reviewed this manuscript before submission.

\section{REFERENCES}

1. Mueller SN, Mackay LK. Tissue-resident memory T cells: local specialists in immune defence. Nat Rev Immunol. (2016) 16:79-89. doi: 10.1038/nri.2015.3

2. Schenkel JM, Masopust D. Tissue-resident memory T cells. Immunity. (2014) 41:886-97. doi: 10.1016/j.immuni.2014.12.007

3. Iijima N, Iwasaki A. Tissue instruction for migration and retention of TRM cells. Trends Immunol. (2015) 36:556-64. doi: 10.1016/j.it.2015.07.002

4. Park SL, Zaid A, Hor JL, Christo SN, Prier JE, Davies B, et al. Local proliferation maintains a stable pool of tissue-resident memory $\mathrm{T}$ cells after antiviral recall responses. Nat Immunol. (2018) 19:18391. doi: 10.1038/s41590-017-0027-5

5. Hombrink P, Helbig C, Backer RA, Piet B, Oja AE, Stark R, et al. Programs for the persistence, vigilance and control of human $\mathrm{CD} 8(+)$ lung-resident memory T cells. Nat Immunol. (2016) 17:1467-78. doi: 10.1038/ni.3589

6. Stary G, Olive A, Radovic-Moreno AF, Gondek D, Alvarez D, Basto PA, et al. VACCINES. a mucosal vaccine against Chlamydia trachomatis generates two waves of protective memory T cells. Science. (2015) 348:aaa8205. doi: 10.1126/science.aaa8205

7. Shin H. Formation and function of tissue-resident memory T cells during viral infection. Curr Opin Virol. (2018) 28:61-67. doi: 10.1016/j.coviro.2017.11.001

8. Salama NR, Hartung ML, Muller A. Life in the human stomach: persistence strategies of the bacterial pathogen Helicobacter pylori. Nat Rev Microbiol. (2013) 11:385-99. doi: 10.1038/nrmicro3016

9. Czinn SJ, Blanchard T. Vaccinating against Helicobacter pylori infection. Nat Rev Gastroenterol Hepatol. (2011) 8:133-40. doi: 10.1038/nrgastro.2011.1

10. Sutton P, Chionh YT. Why can't we make an effective vaccine against Helicobacter pylori? Expert Rev Vaccines. (2013) 12:43341. doi: $10.1586 /$ erv. 13.20

11. Baldari CT, Lanzavecchia A, Telford JL. Immune subversion by Helicobacter pylori. Trends Immunol. (2005) 26:199-207. doi: 10.1016/j.it.2005.01.007

12. Akhiani AA, Schon K, Franzen LE, Pappo J, Lycke N. Helicobacter pylorispecific antibodies impair the development of gastritis, facilitate bacterial colonization, and counteract resistance against infection. J Immunol. (2004) 172:5024-33. doi: 10.4049/jimmunol.172.8.5024

13. Ermak TH, Giannasca PJ, Nichols R, Myers GA, Nedrud J, Weltzin R, et al. Immunization of mice with urease vaccine affords protection against Helicobacter pylori infection in the absence of antibodies and is mediated by MHC class II-restricted responses. J Exp Med. (1998) 188:2277-88.

\section{FUNDING}

This research was sponsored by the National key R\&D Program of China (No. 2017YFD0400303), National Natural Science Foundation of China (No. 81502970), the Priority Academic Program Development (PAPD) of Jiangsu Higher Education Institutions and Postgraduate Research \& Practice Innovation Program of Jiangsu Province.

\section{ACKNOWLEDGMENTS}

We thank Dr. Zhaohui Wang, Dr. Linxi Zhang and Dr. Gao Chao for their help for revision. We would like to thank AJE (https://www.aje.com) for English language editing.

\section{SUPPLEMENTARY MATERIAL}

The Supplementary Material for this article can be found online at: https://www.frontiersin.org/articles/10.3389/fimmu. 2019.01115/full\#supplementary-material

14. Tan Z, Liu W, Liu H, Li C, Zhang Y, Meng X, et al. Oral Helicobacter pylori vaccine-encapsulated acid-resistant HP55/PLGA nanoparticles promote immune protection. Eur J Pharm Biopharm. (2017) 111:33-43. doi: 10.1016/j.ejpb.2016.11.007

15. Moyat M, Bouzourene H, Ouyang W, Iovanna J, Renauld JC, Velin D. IL22 -induced antimicrobial peptides are key determinants of mucosal vaccineinduced protection against H. pylori in mice. Mucosal Immunol. (2017) 10:271-81. doi: 10.1038/mi.2016.38

16. Velin D, Narayan S, Bernasconi E, Busso N, Ramelli G, Maillard $\mathrm{MH}$, et al. PAR2 promotes vaccine-induced protection against Helicobacter infection in mice. Gastroenterology. (2011) 141:1273-82, 1282 el. doi: 10.1053/j.gastro.2011.06.038

17. Guo L, Yin R, Liu K, Lv X, Li Y, Duan X, et al. Immunological features and efficacy of a multi-epitope vaccine CTB-UE against $\mathrm{H}$. pylori in BALB/c mice model. Appl Microbiol Biotechnol. (2014) 98:3495507. doi: 10.1007/s00253-013-5408-6

18. O'Connor A, Vaira D, Gisbert JP, O'Morain C. Treatment of Helicobacter pylori infection 2014. Helicobacter. (2014) 19(Suppl 1):38-45. doi: 10.1111/hel.12163

19. Liu W, Tan Z, Xue J, Luo W, Song H, Lv X, et al. Therapeutic efficacy of oral immunization with a non-genetically modified Lactococcus lactis-based vaccine CUE-GEM induces local immunity against Helicobacter pylori infection. Appl Microbiol Biotechnol. (2016) 100:6219-29. doi: 10.1007/s00253-016-7333-y

20. Song H, Lv X, Yang J, Liu W, Yang H, Xi T, et al. A novel chimeric flagellum fused with the multi-epitope vaccine CTB-UE prevents Helicobacter pyloriinduced gastric cancer in a BALB/c mouse model. Appl Microbiol Biotechnol. (2015) 99:9495-502. doi: 10.1007/s00253-015-6705-Z

21. Ng GZ, Sutton P. An optimised perfusion technique for extracting murine gastric leukocytes. J Immunol Methods. (2015) 427:126-9. doi: 10.1016/j.jim.2015.10.004

22. Liu W, Tan Z, Liu H, Zeng Z, Luo S, Yang H, et al. Nongenetically modified Lactococcus lactis-adjuvanted vaccination enhanced innate immunity against Helicobacter pylori. Helicobacter. (2017) 22:e12426 doi: 10.1111/ hel.12426

23. Davies B, Prier JE, Jones CM, Gebhardt T, Carbone FR, Mackay LK. Cutting edge: tissue-resident memory $\mathrm{t}$ cells generated by multiple immunizations or localized deposition provide enhanced immunity. J Immunol. (2017) 198:2233-7. doi: 10.4049/jimmunol.1601367 
24. Bergsbaken T, Bevan MJ. Proinflammatory microenvironments within the intestine regulate the differentiation of tissue-resident CD8(+) $\mathrm{T}$ cells responding to infection. Nat Immunol. (2015) 16:406-14. doi: 10.1038/ni.3108

25. Bergsbaken T, Bevan MJ, Fink PJ. Local inflammatory cues regulate differentiation and persistence of CD8 $(+)$ tissue-resident memory T cells. Cell Rep. (2017) 19:114-24. doi: 10.1016/j.celrep.2017.03.031

26. Ijima N, Iwasaki A. T cell memory. a local macrophage chemokine network sustains protective tissue-resident memory CD4 T cells. Science. (2014) 346:93-8. doi: 10.1126/science. 1257530

27. Collins N, Jiang X, Zaid A, Macleod BL, Li J, Park CO, et al. Skin CD4(+) memory $\mathrm{T}$ cells exhibit combined cluster-mediated retention and equilibration with the circulation. Nat Commun. (2016) 7:11514. doi: $10.1038 /$ ncomms 11514

28. DeLyria ES, Redline RW, Blanchard TG. Vaccination of mice against H pylori induces a strong Th-17 response and immunity that is neutrophil dependent. Gastroenterology. (2009) 136:247-56. doi: 10.1053/j.gastro.2008.09.017

29. Velin D, Favre L, Bernasconi E, Bachmann D, Pythoud C, Saiji E, et al. Interleukin-17 is a critical mediator of vaccine-induced reduction of Helicobacter infection in the mouse model. Gastroenterology. (2009) 136:2237-46 e1. doi: 10.1053/j.gastro.2009.02.077

30. Zeng M, Mao XH, Li JX, Tong WD, Wang B, Zhang YJ, et al. Efficacy, safety, and immunogenicity of an oral recombinant Helicobacter pylori vaccine in children in China: a randomised, double-blind, placebo-controlled, phase 3 trial. Lancet. (2015) 386:1457-64. doi: 10.1016/S0140-6736(15)60310-5

31. Sigal M, Logan CY, Kapalczynska M, Mollenkopf HJ, Berger H, Wiedenmann B, et al. Stromal R-spondin orchestrates gastric epithelial stem cells and gland homeostasis. Nature. (2017) 548:451-5. doi: 10.1038/nature23642

32. Maixner F, Krause-Kyora B, Turaev D, Herbig A, Hoopmann MR, Hallows JL, et al. The 5300-year-old Helicobacter pylori genome of the Iceman. Science. (2016) 351:162-5. doi: 10.1126/science.aad2545

33. Uemura N, Okamoto S, Yamamoto S, Matsumura N, Yamaguchi S, Yamakido $\mathrm{M}$, et al. Helicobacter pylori infection and the development of gastric cancer. N Engl J Med. (2001) 345:784-9. doi: 10.1056/NEJMoa001999

34. Li H, Yang T, Liao T, Debowski AW, Nilsson HO, Fulurija A, et al. The redefinition of Helicobacter pylori lipopolysaccharide Oantigen and core-oligosaccharide domains. PLoS Pathog. (2017) 13:e1006280. doi: 10.1371/journal.ppat.1006280

35. Gaddy JA, Radin JN, Cullen TW, Chazin WJ, Skaar EP, Trent MS, Algood HM. Helicobacter pylori resists the antimicrobial activity of calprotectin via lipid a modification and associated biofilm formation. MBio. (2015) 6:e0134915. doi: 10.1128/mBio.01349-15

36. Velin D, Bachmann D, Bouzourene H, Michetti P. Mast cells are critical mediators of vaccine-induced Helicobacter clearance in the mouse model. Gastroenterology. (2005) 129:142-55. doi: 10.1053/j.gastro.2005.04.010

37. Hitzler I, Oertli M, Becher B, Agger EM, Muller A. Dendritic cells prevent rather than promote immunity conferred by a helicobacter vaccine using a mycobacterial adjuvant. Gastroenterology. (2011) 141:186-96, 196 e1. doi: 10.1053/j.gastro.2011.04.009

38. Sutton P, Danon SJ, Walker M, Thompson LJ, Wilson J, Kosaka $\mathrm{T}$, et al. Post-immunisation gastritis and Helicobacter infection in the mouse: a long term study. Gut. (2001) 49:467-73. doi: 10.1136/ gut.49.4.467

39. Beura LK, Hamilton SE, Bi K, Schenkel JM, Odumade OA, Casey KA, et al. Normalizing the environment recapitulates adult human immune traits in laboratory mice. Nature. (2016) 532:512-6. doi: 10.1038/nature17655

40. Steinert EM, Schenkel JM, Fraser KA, Beura LK, Manlove LS, Igyarto $\mathrm{BZ}$, et al. Quantifying memory CD8 $\mathrm{T}$ cells reveals regionalization of immunosurveillance. Cell. (2015) 161:737-49. doi: 10.1016/j.cell.2015.03.031

41. Beura LK, Mitchell JS, Thompson EA, Schenkel JM, Mohammed J, Wijeyesinghe S, et al. Intravital mucosal imaging of CD8(+) resident memory $\mathrm{T}$ cells shows tissue-autonomous recall responses that amplify secondary memory. Nat Immunol. (2018) 19:173-82. doi: 10.1038/s41590-017-0029-3

42. Zaid A, Mackay LK, Rahimpour A, Braun A, Veldhoen M, Carbone FR, et al. Persistence of skin-resident memory T cells within an epidermal niche. Proc Natl Acad Sci USA. (2014) 111:5307-12. doi: 10.1073/pnas.1322292111

43. Turner DL, Bickham KL, Thome JJ, Kim CY, D'Ovidio F, Wherry EJ, et al. Lung niches for the generation and maintenance of tissue-resident memory $\mathrm{T}$ cells. Mucosal Immunol. (2014) 7:501-10. doi: 10.1038/mi.2013.67

44. Park CO, Fu X, Jiang X, Pan Y, Teague JE, Collins N, et al. Staged development of long-lived T-cell receptor alphabeta TH17 resident memory T-cell population to Candida albicans after skin infection. J Allergy Clin Immunol. (2017) 142:647-62. doi: 10.1016/j.jaci.2017.09.042

45. Glennie ND, Yeramilli VA, Beiting DP, Volk SW, Weaver CT, Scott P. Skinresident memory CD4+ T cells enhance protection against Leishmania major infection. J Exp Med. (2015) 212:1405-14. doi: 10.1084/jem.20142101

46. Teijaro JR, Turner D, Pham Q, Wherry EJ, François L, Farber DL. Cutting edge: tissue-retentive lung memory CD4 T cells mediate optimal protection to respiratory virus infection. J Immunol. (2011) 187:55104. doi: 10.4049/jimmunol.1102243

47. Schenkel JM, Fraser KA, Beura LK, Pauken KE, Vezys V, Masopust D. T cell memory. resident memory CD8 T cells trigger protective innate and adaptive immune responses. Science. (2014) 346:98-101. doi: 10.1126/science.1254536

48. Abramson A, Caffarel-Salvador E, Khang M, Dellal D, Silverstein D, Gao Y, et al. An ingestible self-orienting system for oral delivery of macromolecules. Science. (2019) 363:611-5. doi: 10.1126/science.aau2277

49. Pan Y, Tian T, Park CO, Lofftus SY, Mei S, Liu X, et al. Survival of tissueresident memory $\mathrm{T}$ cells requires exogenous lipid uptake and metabolism. Nature. (2017) 543:252-6. doi: 10.1038/nature21379

Conflict of Interest Statement: The authors declare that the research was conducted in the absence of any commercial or financial relationships that could be construed as a potential conflict of interest.

Copyright (C) 2019 Liu, Zeng, Luo, Hu, Xu, Huang, Zheng, Sundberg, Xi and Xing. This is an open-access article distributed under the terms of the Creative Commons Attribution License (CC BY). The use, distribution or reproduction in other forums is permitted, provided the original author(s) and the copyright owner(s) are credited and that the original publication in this journal is cited, in accordance with accepted academic practice. No use, distribution or reproduction is permitted which does not comply with these terms. 